$17^{\text {th }}$ International Congress of Metrology, 03003 (2015)

DOI: $10.1051 /$ metrology $/ 201503003$

(C) Owned by the authors, published by EDP Sciences, 2015

\title{
Determination of the Blockage Effect on a Thermal Anemometer using a Small Open Jet Wind Tunnel
}

\author{
Stephen R. Rickaby \& David Highton \\ Antech Calibration Services (a member of the Trescal group), Gapton Hall Industrial Estate, Great Yarmouth, \\ Norfolk, NR31 ONN, UK
}

ABSTRACT: When an object, specifically an anemometer, is presented before a wind tunnel the flow field will be altered deflecting the flow around the anemometer creating what is commonly known as the Blockage Effect. Directly comparing a thermal anemometer with a vane anemometer in the same flow field, the velocity measured by the thermal anemometer may be significantly different to that measured by the vane anemometer as a result of blockage. In this paper we consider the blockage created by a thermal anemometer. A simple mathematical model is derived to directly compare the thermal anemometer with a primary standard vane anemometer. The calibration results obtained are compared with those obtained by the manufacturer and an ISO/IEC 17025 accredited laboratory chosen as the Reference Laboratory for the purposes of the paper. We conclude with an analysis of the results, discussing the differences in the measured output and postulating how these results may be unified.

\section{INTRODUCTION}

In many industrial and research sectors the measurement of air velocity is performed using an anemometer. There are several designs of anemometer including: thermal probe, cup and vane. A Pitot tube used in conjunction with a micromanometer is also commonly used as a method of measuring air velocity. The Pitot tube is a mechanical device used for measuring dynamic pressure, from this the velocity of the flow can be determined. In contrast, thermal anemometers derive a velocity measurement from a relationship between temperature and electrical resistance.

A wind tunnel is designed to generate a constant, laminar, uniform velocity. Anemometers are normally calibrated using a wind tunnel. The procedure adopted in this research is to measure the velocity flow field generated by the wind tunnel with a primary standard anemometer. The primary standard is then substituted for the instrument under consideration keeping the velocity of the flow fixed, with the sensor of both instruments located at the same distance from the end of the wind tunnel and in the same orientation.

The flow field will be altered when an object is placed within it, causing pressure to accumulate in the flow direction, creating what is known as the Blockage Effect, see Figure 1.

The blockage effect, , is the sum of solid blockage, $s$, and wake blockage, $w$, i.e. $\epsilon=\epsilon_{s}+\epsilon_{w}$. Solid blockage results from the flow being unable to pass through an object and

hence is a function of the area causing the blockage. As flow passes over a bluff-body it separates at the edges of the body generating regions of lower mean velocity, these regions are termed the wake.

The blockage effect is therefore directly influenced by

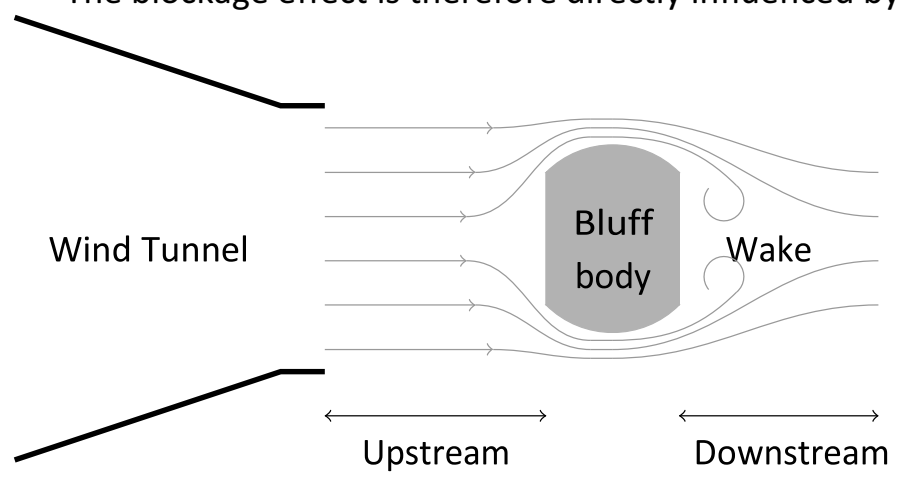

Figure 1: An illustration of the Blockage Effect. The grey lines represent the flow-field, streamlines, around the bluffbody with the arrows denoting the direction of flow.

the physical size of the bluff-body. The bluff-body in this instance is the primary reference standard used to measure the flow field and the anemometer under calibration. The flow field will be significantly altered when using a $6 \mathrm{~mm}$ diameter thermal anemometer in comparison to a vane anemometer with a $100 \mathrm{~mm}$ diameter. If blockage is not taken into consideration then the velocity measured by the thermal anemometer may read differently to that measured by the vane anemometer. 
In this paper we develop a blockage model for a thermal anemometer using a small open jet wind tunnel. The model and design we have chosen is a Dantec 54T35 omnidirectional thermal probe used in conjunction with the Comfortsense Mini 54M95 signal conditioner. For a given velocity this anemometer provides a voltage flow (Maskell 1963) developed the following relation which includes the effects of drag:

$$
\text { Maskell }=\frac{5}{2} C_{D} \frac{S}{C},
$$

\begin{tabular}{ccccccc}
\hline $\begin{array}{c}\text { Reference } \\
\text { velocity }\end{array}$ & $\begin{array}{c}\text { Measured } \\
\text { velocity }\end{array}$ & Error & $\begin{array}{c}\text { Adjusted } \\
\text { velocity }\end{array}$ & Error & Tolerance & $\begin{array}{c}\text { Mach } \\
\text { number }\end{array}$ \\
$v_{r}$ & $v m$ & $v m-v r$ & $v_{b}$ & $v b-v r$ & $\pm(0.6 \%+0.02 \mathrm{~m} / \mathrm{s})$ & \\
$\mathrm{m} / \mathrm{s}$ & $\mathrm{m} / \mathrm{s}$ & $\pm \mathrm{m} / \mathrm{s}$ & $\mathrm{m} / \mathrm{s}$ & $\pm \mathrm{m} / \mathrm{s}$ & $\pm \mathrm{m} / \mathrm{s}$ & \\
\hline 1.55 & 1.62 & 0.07 & 1.54 & -0.01 & 0.03 & 0.01 \\
2.01 & 2.14 & 0.13 & 2.03 & 0.02 & 0.03 & 0.02 \\
5.00 & 5.33 & 0.33 & 5.05 & 0.05 & 0.05 & 0.04 \\
10.08 & 10.70 & 0.62 & 10.14 & 0.06 & 0.08 & 0.09 \\
15.02 & 15.81 & 0.79 & 14.98 & -0.04 & 0.11 & 0.13 \\
20.00 & 21.15 & 1.15 & 20.03 & 0.03 & 0.14 & 0.17 \\
29.95 & 31.43 & 1.48 & 29.77 & -0.18 & 0.20 & 0.27 \\
\hline
\end{tabular}

Table 1: Antech calibration results for the comparison between a Pitot tube and a 100mm vane anemometer. The adjusted velocity is derived by taking blockage into consideration. The Mach number is calculated using the specific ambient

output, thus, in order to establish the velocity a relationship between conditions at each calibration point.

voltage and velocity must first be determined. The blockage model for the Dantec 54T35 omnidirectional thermal probe anemometer will be derived using a Pitot tube and $100 \mathrm{~mm}$ vane anemometer as primary reference standards. In Section 2 we describe the theory and method used in developing the blockage model. Calibration results using the developed model are given in Section 3. In Section 3 we provide also the calibration results of the manufacturer and an ISO/IEC 17025 accredited laboratory. We provide an analysis of the results in Section 4, where the calibration results of the ISO/IEC 17025 accredited laboratory are used as the reference. Finally, in Section 5 a discussion of the results is provided.

\section{CONCEPTUAL MODEL}

As a first model for the calibration of the thermal anemometer we follow the work of (Barlow et al. 1999, Pages 328-366), who derived the simple relation for blockage:

$$
\text { Barlow }=\frac{1}{4} \frac{S}{C},
$$

where $S$ is the frontal area of the object causing blockage and $C$ is the cross-sectional area of the wind tunnel. From eq. (1) we see that blockage is inversely proportional to the cross-sectional area of the nozzle of the open jet wind tunnel. For a three-dimensional bluff-body in a streamline here $C_{D}$ is the drag coefficient. Both these constitutive equations have been extensively referenced in current literature, see for example (Ross and Altman 2011).

A Pitot tube is placed at a distance $h$ from the end of the wind tunnel and calibrated against a vane anemometer as primary standard. If the Pitot tube is now replaced by a thermal anemometer at the same distance $h$, of similar diameter, then from eq. (1) it is postulated that the blockage factor for the Pitot tube and thermal anemometer will be analogous.

The L-type Pitot tube is aligned with the nozzle in the center of the wind tunnel orientated such that the nozzle is

\begin{tabular}{rcccc}
\hline$v_{r}$ & $v_{m}$ & $\frac{1}{2} \rho\left(v_{m}^{2}-v_{r}^{2}\right)$ & \multicolumn{1}{l}{$v_{b}$} & $\frac{1}{2} \rho\left(v_{b}^{2}-v_{r}^{2}\right)$ \\
$\mathrm{m} / \mathrm{s}$ & $\mathrm{m} / \mathrm{s}$ & $\mathrm{Pa}$ & $\mathrm{m} / \mathrm{s}$ & $\mathrm{Pa}$ \\
\hline 1.55 & 1.62 & 0.134 & 1.54 & -0.025 \\
2.01 & 2.14 & 0.317 & 2.03 & 0.040 \\
5.00 & 5.33 & 2.021 & 5.05 & 0.303 \\
10.08 & 10.70 & 7.655 & 10.14 & 0.731 \\
15.02 & 15.81 & 14.337 & 14.98 & -0.764 \\
20.00 & 21.15 & 27.846 & 20.03 & 0.823 \\
29.95 & 31.43 & 53.434 & 29.77 & -6.242 \\
\hline
\end{tabular}

Table 2: Antech calibration results for the comparison between a Pitot tube and a $100 \mathrm{~mm}$ vane anemometer. The results illustrate the difference in pressure before and after blockage has been taken into consideration. 
perpendicular to the flow field. The thermal anemometer is placed in the same orientation as the Pitot tube, i.e. parallel to the direction of flow.

If the wind tunnel has a radius $r$ then the frontal area of the Pitot tube causing the blockage may be approximated by $S_{1} \approx r d_{1}$ where $d_{1}$ is the diameter of the Pitot tube.

The thermal anemometer under test has a removable shroud surrounding the thermal sensor. With the shroud removed there are no apertures or obstructions which may alter the flow field, allowing a direct comparison to be made with the Pitot tube. The frontal area of the thermal anemometer causing blockage is approximated by $S_{2} \approx r d_{2}-\eta$ where $d_{2}$ is the diameter of the shaft of the thermal anemometer and $\eta$ is the difference between the shaft and the sensor. In practice $\left|S_{2}-S_{1}\right| \ll 30 \mathrm{~mm}^{2}$.

From eq. (1) we may assume that the blockage of the thermal anemometer will be linear and remain within the linear regime over the calibration range $0.1 \mathrm{~m} / \mathrm{s}$ to $30.0 \mathrm{~m} / \mathrm{s}$, being the manufacturer's specification for the Dantec 54T35 omnidirectional thermal anemometer. However, linearity may not necessarily follow for a different design of anemometer such as a cup or vane. For means of comparison against the $100 \mathrm{~mm}$ vane we have used a Furness Controls 300mm Pitot tube, type FC065. To achieve stability the blockage factor will be derived by calibrating the Pitot tube over the range $1.55 \mathrm{~m} / \mathrm{s}$ to $29.95 \mathrm{~m} / \mathrm{s}$.

The results of comparing the Pitot tube against the measured velocities $v_{m}$. The blockage correction factor is calculated using least square regression, such that the error between the measured velocities $v_{r}$ and applied velocities $v_{m}$ are minimised. For the values given in Table 1 a blockage correction factor is calculated to be 1.058 . Multiplying this value with the measured velocities $v_{m}$, we derive the adjusted velocities $v_{b}$. The errors, $v_{b}-v_{r}$, are within a tolerance of $\pm(0.6 \%+0.02 \mathrm{~m} / \mathrm{s})$. The derived blockage correction factor is a unique relation between the Pitot tube and primary standard vane anemometer.

If we assume the flow is isentropic then the Mach Number, Ma, to describe this flow may be defined as:

$$
\mathrm{Ma}=v \sqrt{\frac{\rho}{\gamma p}},
$$

where $v$ is the velocity, $\rho$ is the density and $p$ is the pressure. The dimensionless parameter $\gamma$ is the ratio of specific heats, being the specific heat at constant pressure, $c_{p}$, to the specific heat at constant volume, $c_{v}$, i.e. $\gamma=c_{p} / c_{v}$. This dimensionless parameter may be used to determine flow compressibility. If the Mach Number is significantly less than 1 i.e. Mar< 1 then the flow is considered incompressible.

For the thermal anemometer under consideration, the maximal velocity is $30 \mathrm{~m} / \mathrm{s}$, using this velocity under standard reference conditions of: $p=1013.25 \mathrm{mbar}, T$ $=20.0^{\circ} \mathrm{C}, \rho$ is calculated to be $\rho=1.199 \mathrm{~kg} / \mathrm{m}^{3}$. Taking $\gamma=$ 1.4 then from eq. (3) the Mach number is calculated to be $\mathrm{Ma} \approx 0.3<1$. The flow may therefore be described as

\begin{tabular}{|c|c|c|c|c|c|c|c|}
\hline $\begin{array}{c}\text { Reference velocity } \\
\qquad v_{r} \\
\mathrm{~m} / \mathrm{s}\end{array}$ & $\begin{array}{l}\text { Measured } \\
\text { voltage } \\
\text { V }\end{array}$ & $\begin{array}{l}\text { Measurement } \\
\text { uncertainty } \\
\pm \mathrm{m} / \mathrm{s}\end{array}$ & $\begin{array}{c}\text { Equation (4) } \\
\qquad \begin{array}{c}v_{k} \\
\mathrm{~m} / \mathrm{s}\end{array}\end{array}$ & $\begin{array}{r}\text { Error } \\
v k-v r \\
\mathrm{~m} / \mathrm{s}\end{array}$ & $\begin{array}{l}\text { Polynomial } \\
\qquad p 12 \\
\mathrm{~m} / \mathrm{s}\end{array}$ & $\begin{array}{c}\text { Error } \\
v_{p} 12-v_{r} \\
\mathrm{~m} / \mathrm{s}\end{array}$ & $\begin{array}{c}\text { Manufacturer's } \\
\text { tolerance } \\
\pm \mathrm{m} / \mathrm{s}\end{array}$ \\
\hline 0.107 & 0.21615 & 0.12 & 0.052 & $-0.055^{*}$ & 0.107 & $7.03 \times 10^{-8}$ & 0.022 \\
\hline 0.205 & 0.49615 & 0.07 & 0.163 & $-0.042 *$ & 0.205 & $8.66 \times 10^{-8}$ & 0.024 \\
\hline 0.302 & 0.62790 & 0.18 & 0.248 & $-0.054^{*}$ & 0.302 & $9.47 \times 10^{-8}$ & 0.026 \\
\hline 0.505 & 0.89623 & 0.01 & 0.493 & -0.012 & 0.505 & $8.55 \times 10^{-8}$ & 0.030 \\
\hline 0.801 & 1.15004 & 0.02 & 0.825 & 0.024 & 0.801 & $1.01 \times 10^{-8}$ & 0.036 \\
\hline 1.006 & 1.28297 & 0.02 & 1.045 & 0.039 & 1.006 & $6.85 \times 10^{-8}$ & 0.040 \\
\hline 2.008 & 1.74881 & 0.09 & 2.116 & $0.108 *$ & 2.008 & $8.19 \times 10^{-8}$ & 0.060 \\
\hline 6.004 & 2.65526 & 0.04 & 6.134 & 0.130 & 6.004 & $9.37 \times 10^{-8}$ & 0.140 \\
\hline 10.008 & 3.15269 & 0.07 & 9.953 & -0.055 & 10.008 & $8.50 \times 10^{-8}$ & 0.220 \\
\hline 15.020 & 3.61242 & 0.10 & 14.920 & -0.100 & 15.020 & $9.23 \times 10^{-8}$ & 0.320 \\
\hline 19.99 & 3.96950 & 0.14 & 19.97 & -0.02 & 19.99 & $5.90 \times 10^{-8}$ & 0.42 \\
\hline 25.00 & 4.25881 & 0.14 & 24.96 & -0.04 & 25.00 & $5.88 \times 10^{-8}$ & 1.25 \\
\hline 30.02 & 4.51304 & 0.17 & 30.10 & 0.08 & 30.02 & $7.20 \times 10^{-8}$ & 1.50 \\
\hline
\end{tabular}

Table 3: Reference Laboratory calibration results. In columns 4 and 6 we provide the fit obtained from King's Law eq. (4) and a twelfth order polynomial, respectively. All uncertainties are expressed at a level of confidence of approximately 95\%. The asterisk denoting errors outside the manufacturer's specification.

$100 \mathrm{~mm}$ vane anemometer are given in Table 1 . We take the velocity of the $100 \mathrm{~mm}$ vane as the reference velocities, $v_{r}$, and the velocity of the Pitot tube as the incompressible. For completeness the Mach numbers for the comparison of the Pitot tube against the vane 
anemometer are given in Table 1, where we have used actual ambient conditions at each calibration point.

The flow is now considered to be steady, inviscid and incompressible. The Bernoulli equation for the comparison between two points, 1 and 2, along a streamline may be written:

$p_{1}+\frac{1}{2} \rho v_{1}^{2}=p_{2}+\frac{1}{2} \rho v_{2}^{2} \quad$ or $p_{1}-p_{2}=\frac{\rho\left(v_{2}^{2}-v_{1}^{2}\right)}{2}$

where we have assumed no change in elevation.

Applying eq. (4) to the results given in Table 2 we see the blockage factor minimises the pressure difference $p_{1}$ $-p_{2}$, see Table 2 columns 3 and 5 . From eq. (4) it is noted that if $v_{1}=v_{2}$ then $p_{1}=p_{2}$. It is further noted from Bernoulli's equation if the mean velocity of the wake is less then the free flow, as previously described, the pressure difference of the wake must be smaller than the pressure difference of the free flow.

The Reynolds number is a dimensionless constant commonly used to characterise flow, it is defined as the ratio of inertial to viscous forces. The Reynolds number is related to the velocity of the flow through the equation:

$$
\operatorname{Re}=\frac{\rho v d}{\mu_{\text {air }}}
$$

where $\rho$ and $\mu_{\text {air }}$ are the density and viscosity of the fluid, respectively. The quantity $d$ is a characteristic length of the bluff-body, which we take to be the radius of the Pitot tube.

From eq. (4) we see that as velocity increases the Reynolds number increases, thus the size of the wake will increase.

The diameters of the Pitot tube and the thermal anemometer are within $\pm 1.2 \mathrm{~mm}$, with the diameter of the thermal anemometer being larger. Thus, the Reynolds numbers of the Pitot tube and thermal anemometer will be within $\pm 20 \%$. However, the wake will have a different effect on the Pitot tube compared to the omnidirectional thermal anemometer, in this first model, these differences have been neglected.

At standard reference conditions: $\rho=1.199 \mathrm{~kg} / \mathrm{m}^{3}$ and $\mu_{\text {air }}=1.837 \times 10^{-5} \mathrm{~kg} / \mathrm{ms}$. The Reynolds number for

\begin{tabular}{ccccccc}
\hline Reference velocity & Polynomial & Error & Polynomial & Error & Polynomial & Error \\
$v_{r}$ & $v p 6$ & $v p 6-v r$ & $v p 8$ & $v p 8-v r$ & $v p 10$ & $v_{p} 10-v_{r}$ \\
$\mathrm{~m} / \mathrm{s}$ & $\mathrm{m} / \mathrm{s}$ & $\mathrm{m} / \mathrm{s}$ & $\mathrm{m} / \mathrm{s}$ & $\mathrm{m} / \mathrm{s}$ & $\mathrm{m} / \mathrm{s}$ & $\mathrm{m} / \mathrm{s}$ \\
\hline 0.107 & 0.108 & $-0.08 \times 10^{-2}$ & 0.104 & $0.32 \times 10^{-2}$ & 0.107 & $0.03 \times 10^{-3}$ \\
0.205 & 0.209 & $-0.35 \times 10^{-2}$ & 0.227 & $-2.17 \times 10^{-2}$ & 0.209 & $-3.78 \times 10^{-3}$ \\
0.302 & 0.289 & $1.26 \times 10^{-2}$ & 0.282 & $1.95 \times 10^{-2}$ & 0.295 & $6.67 \times 10^{-3}$ \\
0.505 & 0.514 & $-0.87 \times 10^{-2}$ & 0.492 & $1.29 \times 10^{-2}$ & 0.510 & $-5.15 \times 10^{-3}$ \\
0.801 & 0.809 & $-0.83 \times 10^{-2}$ & 0.812 & $-1.08 \times 10^{-2}$ & 0.802 & $-0.50 \times 10^{-3}$ \\
1.006 & 1.004 & $0.15 \times 10^{-2}$ & 1.020 & $-1.38 \times 10^{-2}$ & 1.002 & $3.80 \times 10^{-3}$ \\
2.008 & 1.991 & $1.65 \times 10^{-2}$ & 1.993 & $1.49 \times 10^{-2}$ & 2.010 & $-1.73 \times 10^{-3}$ \\
6.004 & 6.036 & $-3.19 \times 10^{-2}$ & 6.012 & $-0.79 \times 10^{-2}$ & 6.003 & $1.22 \times 10^{-3}$ \\
10.008 & 9.974 & $3.41 \times 10^{-2}$ & 10.008 & $0.05 \times 10^{-2}$ & 10.010 & $-1.62 \times 10^{-3}$ \\
15.020 & 15.014 & $0.61 \times 10^{-2}$ & 15.007 & $1.33 \times 10^{-2}$ & 15.018 & $1.56 \times 10^{-3}$ \\
19.99 & 20.04 & $-4.77 \times 10^{-2}$ & 20.01 & $-1.92 \times 10^{-2}$ & 19.99 & $-1.10 \times 10^{-3}$ \\
25.00 & 24.96 & $4.18 \times 10^{-2}$ & 24.99 & $1.18 \times 10^{-2}$ & 25.00 & $0.45 \times 10^{-3}$ \\
30.02 & 30.03 & $-1.17 \times 10^{-2}$ & 30.02 & $-0.27 \times 10^{-2}$ & 30.02 & $-0.08 \times 10^{-3}$ \\
\hline
\end{tabular}

Table 4: Polynomial fit to the calibration results of the Reference Laboratory.

\begin{tabular}{ccccccc}
\hline $\begin{array}{c}\text { Reference velocity } \\
v_{r} \\
\mathrm{~m} / \mathrm{s}\end{array}$ & $\begin{array}{c}\text { Measured } \\
\text { voltage } \\
\mathrm{V}\end{array}$ & $\begin{array}{c}\text { Measurement } \\
\text { uncertainty } \\
\pm \mathrm{m} / \mathrm{s}\end{array}$ & $\begin{array}{c}\text { Fitted Velocity } \\
\mathrm{m} / \mathrm{s}\end{array}$ & $\begin{array}{c}\text { Error } \\
V m-V r \\
\mathrm{~m} / \mathrm{s}\end{array}$ & $\begin{array}{c}\text { Manufacturer's } \\
\text { tolerance } \\
\pm \mathrm{m} / \mathrm{s}\end{array}$ & $\begin{array}{c}E_{n} \\
\text { ratio }\end{array}$ \\
\hline 0.515 & 0.9681 & 0.08 & 0.576 & $0.061^{*}$ & 0.0303 & 0.76 \\
1.011 & 1.2968 & 0.09 & 1.029 & 0.018 & 0.0402 & 0.19 \\
1.994 & 1.7546 & 0.10 & 2.024 & 0.030 & 0.0599 & 0.23 \\
5.955 & 2.6553 & 0.17 & 6.004 & 0.049 & 0.1391 & 0.28 \\
9.873 & 3.1573 & 0.27 & 10.053 & 0.180 & 0.2175 & 0.65 \\
14.733 & 3.6159 & 0.42 & 15.063 & $0.330^{*}$ & 0.3147 & 0.76
\end{tabular}




\begin{tabular}{lllllll}
19.70 & 3.9722 & 0.54 & 20.03 & 0.33 & 0.414 & 0.59 \\
24.86 & 4.2599 & 0.68 & 25.02 & 0.16 & 1.243 & 0.23 \\
30.03 & 4.4990 & 0.82 & 29.73 & -0.30 & 1.501 & 0.36 \\
\hline
\end{tabular}

Table 5: Antech calibration results. The fitted velocities have been derived from the calibration values of the Reference Laboratory. All uncertainties are expressed at a level of confidence of approximately $95 \%$. The asterisk denoting errors outside the manufacturer's specification.

\begin{tabular}{|c|c|c|c|c|c|c|}
\hline $\begin{array}{c}\text { Reference Velocity } \\
\qquad v_{r} \\
\mathrm{~m} / \mathrm{s}\end{array}$ & $\begin{array}{l}\text { Measured } \\
\text { voltage } \\
\text { V }\end{array}$ & $\begin{array}{l}\text { Measurement } \\
\text { uncertainty } \\
\pm \mathrm{m} / \mathrm{s}\end{array}$ & $\begin{array}{l}\text { Fitted Velocity } \\
\qquad \begin{array}{c}v_{f} \\
\mathrm{~m} / \mathrm{s}\end{array}\end{array}$ & $\begin{array}{c}\text { Error } \\
v f-v r \\
\mathrm{~m} / \mathrm{s}\end{array}$ & $\begin{array}{c}\text { Manufacturer's } \\
\text { tolerance } \\
\pm \mathrm{m} / \mathrm{s}\end{array}$ & $\begin{array}{c}E_{n} \\
\text { ratio }\end{array}$ \\
\hline 0.095 & 0.3415 & not quoted & 0.087 & -0.009 & 0.0219 & - \\
\hline 0.202 & 0.5661 & not quoted & 0.258 & $0.057 *$ & 0.0240 & - \\
\hline 0.284 & 0.7013 & not quoted & 0.352 & $0.068 *$ & 0.0257 & - \\
\hline 0.514 & 0.9864 & not quoted & 0.596 & $0.082 *$ & 0.0303 & - \\
\hline 0.811 & 1.2274 & not quoted & 0.916 & $0.105 *$ & 0.0362 & - \\
\hline 2.446 & 1.9669 & 0.02 & 2.671 & $0.225 *$ & 0.0689 & 2.52 \\
\hline 6.123 & 2.7390 & 0.03 & 6.574 & $0.451 *$ & 0.1425 & 7.97 \\
\hline 10.28 & 3.2498 & 0.06 & 10.96 & $0.68 *$ & 0.226 & 7.63 \\
\hline 13.03 & 3.5121 & 0.07 & 13.81 & 0.78 & 0.281 & 6.29 \\
\hline 16.39 & 3.7823 & 0.09 & 17.24 & 0.85 & 0.348 & 6.19 \\
\hline 20.25 & 4.0469 & 0.11 & 21.24 & 0.99 & 1.013 & 5.47 \\
\hline 25.32 & 4.3171 & 0.14 & 26.11 & 0.79 & 1.266 & 3.97 \\
\hline 30.75 & 4.5619 & 0.17 & 30.75 & 0.00 & 1.538 & 0.00 \\
\hline
\end{tabular}

Table 6: Manufacturer's calibration results. The fitted velocities have been derived from the calibration values of the Reference Laboratory. All uncertainties are expressed at a level of confidence of approximately 95\%. The asterisk denoting errors outside the manufacturer's specification. 


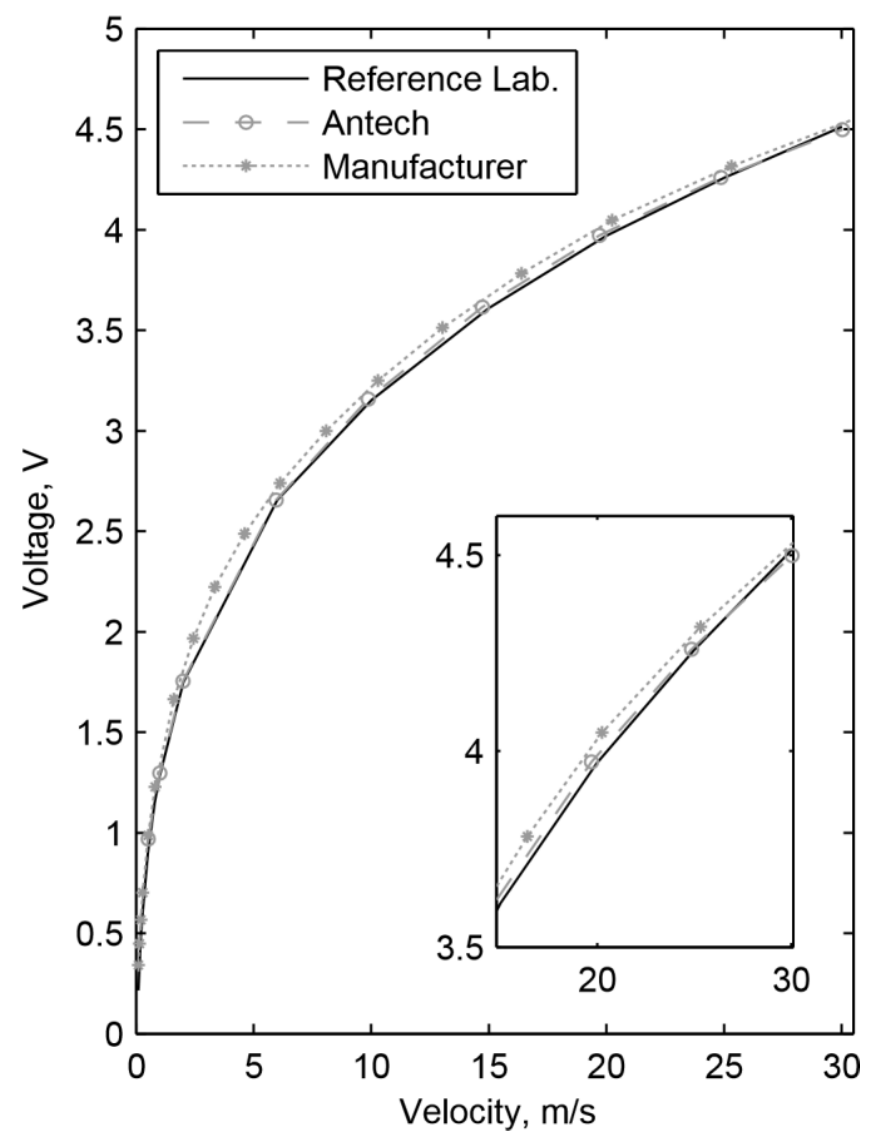

Figure 2: Graphs of the displayed voltages at given velocities as calculated by the manufacturer, the Reference Laboratory and Antech Laboratory, for ease of comparison we have fitted a solid curve through the Reference Laboratory results. The subfigure shows the close similarity in the calibration results between $15 \mathrm{~m} / \mathrm{s}$ and $30 \mathrm{~m} / \mathrm{s}$.

the thermal anemometer under consideration is given by $\operatorname{Re}_{\text {thermal }} \approx 3.916 \times 10^{1} v$. For $0.1 \leq v \leq 30$ we have $1.9 \times$ $10^{3} \leq R e_{\text {thermal }} \leq 1.2 \times 10^{5}$. From these values we would expect boundary layer characteristics, where viscous effects are confined to a thin region near the front surface of the thermal anemometer with the wake region being directly behind it, as shown in Figure 1 . The velocity

We use the blockage model developed in Section 2 to calibrate the Dantec 54T35 omnidirectional thermal probe anemometer with the Comfortsense Mini 54M95 signal conditioner. The results of this calibration are given in Table 5. For completeness we have included also the results of the Reference Laboratory and the manufacturer in Tables 3 and 6, respectively.

In Figure 2 we provide a plot of the calibration results comparing voltages at given velocities for the manufacturer, the Reference Laboratory and Antech's Laboratory. For ease of comparison we have fitted a solid curve through the Reference Laboratory results. gradient in the boundary layer and wake regions will be larger than that in the remainder of the free flow.

A vane anemometer is more obstructive to the flow field then either a thermal or Pitot tube creating an irregular flow field in its surrounding region, see (Care and Arenas 2014). The flow field of a vane anemometer is therefore harder to define. In this work the vane anemometer is used to calibrate both the Pitot tube and thermal anemometer we therefore assume blockage associated with the vane anemometer will affect both instruments under calibration equally.

For the thermal anemometer under consideration the voltage, $E$, varies over the range $0 \leq E \leq 5$, this voltage is related non-linearly to the velocity, $v$, which varies over the range $0.1 \leq v \leq 30$. To limit the errors between these two parameters it is advantageous to develop a constitutive relation of the form:

$$
v=f(E) .
$$

When calibrating constant temperature anemometers several authors use a version of King's law (King 1914)[eq. $\sqrt{ }$

(80)] $E_{2}=A_{1}+A_{2} v$, where $A_{1}$ and $A_{2}$ are constants.

This law may be rewritten,

$$
v=a_{1}-a_{2} E^{2}+a_{3} E^{2}
$$

where $a_{1}, a_{2}$ and $a_{3}$ are constants. Table 3 illustrates the fit in applying this equation to the calibration results produced by the Reference Laboratory. We have used least squares regression analysis to obtain values for the constants. For completeness we have included also the fitted values when using a twelfth order polynomial. In Table 3 the asterisk $(*)$ denotes errors outside the manufacturer's specification, being $\pm 2 \%$ of reading \pm 0.02 $\mathrm{m} / \mathrm{s}$ in the range $0.2 \mathrm{~m} / \mathrm{s}$ to $20 \mathrm{~m} / \mathrm{s}$ and $\pm 5 \%$ of reading in the range $20 \mathrm{~m} / \mathrm{s}$ to $30 \mathrm{~m} / \mathrm{s}$.

As can be seen from Table 3 the fit obtained using the twelfth order polynomial is significantly more accurate

ANALYSIS

The results of the ISO/IEC 17025 accredited laboratory are used as the reference. Using the method of least squares regression the difference between the calibration results of the Reference Laboratory and Antech Laboratory are given in Table 5 where the average error $v_{m}-v_{r}$ over the calibration range of $0.5 \mathrm{~m} / \mathrm{s}$ to $30.0 \mathrm{~m} / \mathrm{s}$ is estimated to be $0.19 \mathrm{~m} / \mathrm{s}$. Similarly, the difference between the calibration results of the Reference Laboratory and manufacturer are given in Table 6 with the average error, $v_{f}-v_{r}$, over the same calibration range is estimated to be $0.41 \mathrm{~m} / \mathrm{s}$. 
than the fit obtained using King's equation. (George et al. 1989) found also a polynomial fit more accurate over the full velocity range.

In Table 4 we provide the resulting error when using a polynomial with 6,8 and 10 terms, respectively, to fit the calibration results of the Reference Laboratory. We observe, in general, for an eighth order polynomial below $6 \mathrm{~m} / \mathrm{s}$ the fit achieved is not as good as the sixth order polynomial. Further, the errors at $0.2 \mathrm{~m} / \mathrm{s}$ and $0.3 \mathrm{~m} / \mathrm{s}$ are approximately equal to the manufacturer's specification, thus illustrating the importance of analysing the polynomial fit to calibration data, so as not to introduce unaccounted-for errors.

In Tables 5 and 6 we calculate the normalized error ratio or $E_{n}$ ratio. The $E_{n}$ ratio is the root sum of the squares of the combined uncertainty as given by the equation:

$$
E_{n}=\frac{\sqrt{\left|v_{\text {lab }}-v_{\text {ref }}\right|}}{2}, \text { ref }
$$

were $v_{\text {ref }}$ is the velocity derived from the Reference Laboratory with expanded uncertainties $U_{\text {ref }}$ and $v_{\text {lab }}$ is the velocity of the laboratory under consideration with expanded uncertainties $U_{\text {lab. The }} E_{n}$ ratio provides a measure of the compatibility of the results, for satisfactory results we require $E_{n} \leq 1$.

As can be seen from the calculated errors and $E_{n}$ ratios the basic model developed here provides good agreement with the results of the Reference Laboratory.

In Tables 5 and 6 values denoted with an asterisk are outside the Manufacturer's specification, several calibration points do not fall within this specification, demonstrating the significance of blockage modelling.

\section{CONCLUSIONS AND FUTURE WORK}

It has been shown that the simple blockage model developed in Section 2 provides good agreement with the Reference Laboratory. This confirms the premise of the postulation that the blockage factor between the Pitot tube and this particular style of thermal anemometer are similar. If the sensor of the thermal anemometer had been protected by a shroud then such close agreement may not have been achieved.

A limitation in using the approach of directly comparing a Pitot tube with a $100 \mathrm{~mm}$ vane anemometer to develop a blockage model, means we are unable to differentiate the individual effect of solid blockage, $s$, and wake blockage, $w$, on the measurement of the velocity flow field.

Through this continued study we aim to minimise the uncertainty of measurement introduced by the blockage effect. We aim to develop our blockage theory further using Particle Image Velocimetry (PIV). PIV is a technique that does not alter the flow field, it works by tracking particles that lie on a common plane, through this the structure of the flow field can be determined. PIV used in conjunction with a large open jet wind tunnel enables the PIV system and the anemometer under consideration to be placed in the same flow field. The flow field will be large enough for the PIV system not to be influenced by blockage generated by the anemometer under consideration, enabling accurate measurement of the flow velocity to be determined. By employing a larger diameter wind tunnel, from the theory supporting eq. (1) we would expect to observe inverse-square decay, $1 / R^{2}$, of the blockage effect, where $R$ is the multiple by which the radius of the wind tunnel has increased, i.e. doubling the radius of the wind tunnel would quarter the blockage effect.

\section{REFERENCES}

Barlow, J. B., W. H. Rae and A. Pope (1999). Low Speed Wind Tunnel Testing. Third Edition, John Wiley and Sons, Inc..

Maskell, E. C. (1963). A theory of the blockage effects on bluff bodies and stalled wings in a closed wind tunnel (No. ARC-R/M-3400). Aeronautical Research Council London (United Kingdom).

Ross, I., and Altman, A. (2011). Wind tunnel blockage corrections: review and application to Savonius vertical-axis wind turbines. J. Wind. Ind. Aerodyn. 99, 523-538.

Care, I., and Arenas, M. (2014). On the impact of anemometer size on the velocity field in a closed wind tunnel. Flow Meas. Instrum.

King, L. S. (1914). On the Convection of Heat from Small Cylinders in a Stream of Fluid: Determination of the Convection Constants of Small Platinum Wires with Applications to Hot-Wire Anemometry. Phil. Trans. $R$. Soc. Lond. 214A, 373-432.

George, W. K., Beuther, P. D. and A. Shabbir (1989). Polynomial Calibration for Hot Wires in Thermally Varying Flows. Exp. Therm. Fluid Sci. 2, 230-235. 